\title{
UMA PROPOSTA PARA A AVALIAÇÃO DA EMPRESA EM CONDIÇÕES DE RISCO COM BASE NO MODELO DE OHLSON*
}

\author{
Edson Ferreira de Oliveira \\ Professor Doutor da PUC-SP \\ Professor Doutor do UNIFIEO-SP \\ E-mail: huguera@uol.com.br

\section{Reinaldo Guerreiro} \\ Professor Titular do Departamento de Contabilidade da FEA-USP \\ E-mail: reiguerr@usp.br

\section{José Roberto Securato} \\ Professor Livre Docente do Departamento de Administração da FEA-USP \\ E-mail: securato@usp.br
}

\section{RESUMO}

O ponto central do trabalho é a proposição de um modelo aleatório de avaliação patrimonial que permita determinar o valor da empresa em condições de risco e definir critérios metodológicos para a quantificação das variáveis utilizadas no processo avaliatório. $\mathrm{O}$ modelo proposto estuda o efeito da aleatoriedade sobre o binômio lucro-patrimônio líquido e suas conseqüências sobre o valor da empresa, utilizando para tanto informações oriundas dos demonstrativos contábeis associadas ao custo do capital próprio. Para atingir o valor das empresas escolhidas, desenvolve-se um estudo exploratório, e a avaliação decorrente, devido às características aleatórias do modelo, conduz a um valor médio e a um desvio-padrão, definindo um intervalo de valores e não um valor único, enriquecendo o processo de avaliação.

Palavras-chave: modelo de Ohlson, avaliação de empresas, condições de risco

\section{ABSTRACT}

This study proposes a random equity valuation model that allows for the valuation of a company in risk conditions and the definition of methodological criteria for quantifying the variables used in the valuation process. The proposed model studies the effect of randomness on the income-equity binomial and its consequences for company value, using information from the financial statements together with the cost of own capital. To obtain the value of the companies that were chosen, an exploratory study is developed and, due to the random characteristics of the model, the resulting valuation leads to an average value and a standard deviation, defining an interval of values and not one single value, which enriches the valuation process.

Keywords: Ohlson's model, company valuation, risk conditions 


\section{INTRODUÇÃO}

Ao longo dos últimos vinte anos vem se construindo uma nova ordem econômica, voltada para a globalização, na qual se destacam a integração dos mercados financeiros, o aparecimento das transnacionais, a desregulamentação do sistema bancário e a desestatização.

Tais processos têm diferentes manifestações na economia, em particular no setor empresarial, onde se apresentam na forma de privatizações, fusões, aquisições e incorporações, onde o fator chave para o processo decisório tem sido o valor da empresa. Este fator é o agente motivador do presente estudo, em torno do qual o trabalho foi desenvolvido.

\section{OBJETIVO DO ESTUDO}

O objetivo do estudo é propor um modelo de avaliação patrimonial da empresa, utilizando variáveis aleatórias, que permita, além da avaliação, definir critérios metodológicos para a quantificação dessas variáveis em condições de risco. A base do modelo são as informações oriundas dos demonstrativos contábeis, ou seja, a demonstração do resultado do exercício e o balanço patrimonial, tratados como variáveis aleatórias.

\section{METODOLOGIA}

O estudo foi desenvolvido com base no método indutivo aplicado ao processo de avaliação da empresa e levou em consideração os seguintes aspectos: (a) observação do fenômeno da valorização, no contexto da empresa, por meio de suas manifestações, traduzidas nas variáveis quantitativas importantes no processo; (b) busca da relação matemático-estatística entre as variáveis quantitativas, dependentes e independentes, procurando determinar um modelo descritivo da valorização da empresa e (c) estudo exploratório com a finalidade de obter a primeira constatação do modelo proposto.

\section{AVALIAÇÃO BASEADA NA COMBINAÇÃO DO LUCRO COM O PATRIMÔNIO LÍQUIDO}

Nos últimos anos vem tomando corpo uma abordagem da análise do valor da empresa que combina medidas de desempenho da empresa com os imperativos da criação de valor. Tal abordagem mescla o rigor teórico do modelo de avaliação baseado no valor presente dos fluxos futuros de dividendos, com o uso de conceitos de contabilidade que traduzem os fatores que impulsionam o valor da empresa, os value drivers, tais como o lucro e o retorno sobre o capital investido. A literatura que embasa essa abordagem está lastreada nos Modelos de GORDON (1956) e de OHLSON (1991). Os conceitos de avaliação desenvolvidos por alguns autores tais como COPELAND et al. (2000), McTAGGART et al. (1994) e STEWART (1991) refletem a influência desta abordagem. LOPES (2001), por sua vez, desenvolve uma abordagem crítica da relevância da informação contábil para a análise do valor no mercado de capitais brasileiro, lançando mão do modelo de Ohlson.

Ohlson, utilizando os conceitos de lucro limpo e lucro residual, explorados por PEASNELL (1982, p. 362-365), foi capaz de descrever as conexões "entre o produto do processo de apropriação de receitas e despesas e o valor das ações de uma firma" (O'HANLON \& REES, 1995, p. 227). O trabalho de Ohlson teve o grande mérito de lançar luz sobre a utilidade dos números contábeis no processo de avaliação, na medida em que estabeleceu a ligação entre o lucro contábil e o valor das ações da empresa, bem como incrementou as discussões sobre o papel da contabilidade e suas limitações para fins de avaliação.

As premissas básicas do modelo de Ohlson podem ser expressas como segue.

a) O preço da ação da empresa é dado pelo valor presente dos dividendos futuros esperados, descontados a uma taxa de retorno requerida:

$$
P_{0}=\sum_{t=1}^{\infty} \frac{d_{t}}{\left(1+K_{e}\right)^{t}} \quad ; \text { onde }
$$

$\mathrm{P}_{0}=$ preço atual da ação

$\mathrm{d}_{\mathrm{t}}=$ dividendos líquidos pagos no final do período $t$ $\mathrm{k}_{\mathrm{e}}$ = taxa de desconto, assumida constante, igual ao custo de capital próprio

b) Considerando $L_{t}$ o lucro limpo da empresa no período $t$, que inclui no lucro contábil do período todas as alterações não derivadas de injeções ou distribuições de capital, pode-se anotar: 


$$
(P L)_{t}=(P L)_{t-1}+L_{t}-d_{t} \quad \text {; onde }
$$

$(P L)_{t}=$ valor do patrimônio líquido contábil no final do período $t$

$(P L)_{t-1}=$ valor do patrimônio líquido contábil no final do período $\mathrm{t}-1$

c) A relação do lucro residual, isto é, lucro ajustado pelo custo do capital próprio é definida por:

$$
L_{t}^{a}=L_{t}-k_{e}(P L)_{t-1}
$$

d) Substituindo a relação do lucro limpo na relação do lucro residual, obtém-se:

$$
d_{t}=L^{a}+\left(1+k_{e}\right) \cdot(P L)_{t-1}-(P L)_{t}
$$

e) A relação anterior, substituída na expressão do preço da ação, conduz a:

$$
P_{0}=(P L)_{0}+\sum_{t=1}^{\infty} \frac{L_{t}^{a}}{\left(1+K_{e}\right)^{t}}
$$

O valor patrimonial da empresa é, portanto, o resultado da soma do seu patrimônio líquido com o valor presente dos seus lucros residuais futuros esperados.

O modelo de Ohlson poderá melhor descrever a realidade se o valor patrimonial da empresa e o lucro residual assumirem caráter aleatório. A aleatoriedade das variáveis está associada às influências dos fatores conjunturais e específicos da empresa. A consideração do caráter aleatório do valor patrimonial da empresa e dos lucros residuais que o determinam permitirá quantificar o risco presente na avaliação da empresa. Isto significa que o problema da avaliação da empresa será, em última análise, o de determinar as características da variável aleatória dependente valor patrimonial, indicada por $\tilde{\mathrm{P}}_{0}$.

\section{MODELO DO VALOR PATRIMONIAL ALEATÓRIO}

A modelagem ora proposta se beneficia dos estudos, análises e parametrizações apresentadas por OLIVEIRA (2001).

Considera-se a seqüência de valores de lucros residuais futuros da empresa representada por:

$$
\left|\tilde{L^{a}}{ }_{t}\right|_{t=1, \infty}=\left\langle\tilde{L}_{1}^{\tilde{a}}, \tilde{L}_{2}^{\tilde{a}}, \tilde{L^{a}}, \ldots \tilde{L_{\infty}}{ }_{\infty}\right|
$$

onde cada lucro residual futuro na data té uma variável aleatória expressa por:

$\tilde{L}_{1}^{\tilde{a}}: D_{t}\left(L_{\mu t}^{a}, L_{S t}^{a}\right)$

ou seja, uma distribuição de probabilidades $D_{t}$, com média $L_{\mu_{t}}^{a}$ e desvio padrão $L_{S_{t}}^{a}$

A seqüência de valores de lucros residuais pode ser tratada de forma análoga à proposta por COPELAND et al. (2000, p. 126), ou seja: (i) projetar os lucros residuais aleatórios da empresa por cem anos e trazê-los a valor presente, não se preocupando com o que vem depois, porque seu valor descontado seria desprezível; ou (ii) separar os lucros residuais aleatórios em dois períodos: um período de projeção explícita e outro período posterior; o valor presente dos lucros residuais seria igual à soma dos valores presentes dos lucros residuais desses dois períodos.

O lucro residual da empresa após o período de projeção explícita, determinado no final desse período, será denominado lucro residual terminal.

Isto posto, a seqüência de lucros residuais da empresa pode ser representada da seguinte forma:

$$
\mid \tilde{L}_{t}{ }_{t=1, N}=\left\langle\tilde{L}_{1}, \tilde{L}_{2}^{\tilde{a}}, \tilde{L}_{3}^{a}, \ldots \tilde{L}_{N}^{a}, \tilde{L}_{T N}\right|
$$

onde $\mathrm{L}_{\mathrm{TN}}^{\mathrm{a}}=$ lucrõ residual terminal da empresa na data $\mathrm{N}$, correspondente ao final do período explícito.

A taxa de desconto a ser utilizada é $k_{e}$. A média e o desvio-padrão do valor patrimonial $P_{0}$ da seqüência dada, considerado uma variável aleatória, serão a seguir determinados.

$$
\begin{array}{cccc}
\tilde{L}_{1}^{\mathrm{a}} & \tilde{L}_{2}^{\mathrm{a}} & \tilde{L}_{3}^{\mathrm{a}} & \left(\tilde{L}_{\mathrm{N}}{ }^{\mathrm{a}}+\tilde{L}_{\mathrm{TN}}^{\mathrm{a}}\right)
\end{array}
$$

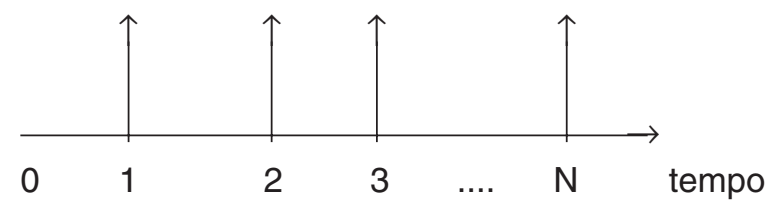

Como:

$\mathrm{P}_{0}=(\mathrm{PL})_{0}+\frac{\mathrm{L}_{1}^{\mathrm{a}}}{\left(1+\mathrm{k}_{\mathrm{e}}\right)}+\frac{\mathrm{L}_{2}^{\mathrm{a}}}{\left(1+\mathrm{K}_{\mathrm{e}}\right)^{2}}+\ldots+\frac{\mathrm{L}_{\mathrm{N}}^{\mathrm{a}}+\mathrm{L}_{\mathrm{TN}}^{\mathrm{a}}}{\left(1+\mathrm{K}_{\mathrm{e}}\right)^{\mathrm{N}}} ;$ decorre 
- Média de $\mathbf{P}_{0}$

$$
\begin{aligned}
& \mathrm{P}_{\mu_{0}}=E\left[\mathrm{P}_{0}\right]=\mathrm{E}\left[(\mathrm{PL})_{\mathrm{C}}+\frac{\mathrm{L}_{1}^{\mathrm{a}}}{\left(1+\mathrm{k}_{\mathrm{e}}\right)}+\frac{\mathrm{L}_{2}^{\mathrm{a}}}{\left(1+\mathrm{k}_{\mathrm{e}}\right)^{2}}+\cdots+\frac{\mathrm{L}_{\mathrm{N}}^{\mathrm{a}}+\mathrm{L}_{\mathrm{TN}}^{\mathrm{a}}}{\left(1+\mathrm{k}_{\mathrm{e}}\right)^{\mathrm{N}}}\right] \\
& \mathrm{P}_{\mu_{0}}=(\mathrm{PL})_{0}+\frac{\mathrm{L}_{\mu_{1}}^{\mathrm{a}}}{\left(1+\mathrm{k}_{\mathrm{e}}\right)}+\frac{\mathrm{L}_{\mu_{2}}^{\mathrm{a}}}{\left(1+\mathrm{k}_{\mathrm{e}}\right)^{2}}+\cdots+\frac{\mathrm{L}_{\mu_{\mathrm{N}}}^{\mathrm{a}}+\mathrm{L}_{\mu_{\mathrm{TN}}}^{\mathrm{a}}}{\left(1+\mathrm{k}_{\mathrm{e}}\right)^{\mathrm{N}}} ; \mathrm{Ou} \\
& \mathrm{P}_{\mu_{0}}=(\mathrm{PL})_{\mathrm{C}}+\sum_{\mathrm{t}=1}^{\mathrm{N}} \frac{\mathrm{L}_{\mu_{\mathrm{t}}}^{\mathrm{a}}}{\left(1+\mathrm{k}_{\mathrm{e}}\right)^{\mathrm{t}}}+\frac{\mathrm{L}_{\mu_{\mathrm{TN}}}^{\mathrm{a}}}{\left(1+\mathrm{k}_{\mathrm{e}}\right)^{\mathrm{N}}}
\end{aligned}
$$

- Desvio-padrão de $\mathrm{P}_{0}$

$$
\begin{aligned}
& \mathrm{P}_{\mathrm{S}_{0}}^{2}=\mathrm{S}^{2}\left[\mathrm{P}_{0}\right]=\mathrm{S}^{2}\left[(\mathrm{PL})_{0}+\frac{\mathrm{L}_{1}^{\mathrm{a}}}{\left(1+\mathrm{k}_{\mathrm{e}}\right)}+\frac{\mathrm{L}_{2}^{\mathrm{a}}}{\left(1+\mathrm{k}_{\mathrm{e}}\right)^{2}}+\cdots+\frac{\mathrm{L}_{\mathrm{N}}^{\mathrm{a}}+\mathrm{L}_{\mathrm{TN}}^{\mathrm{a}}}{\left(1+\mathrm{k}_{\mathrm{e}}\right)^{\mathrm{N}}}\right] \\
& \mathrm{P}_{\mathrm{S}_{0}}^{2}=\frac{\left(\mathrm{L}_{\mathrm{S}_{1}}^{\mathrm{a}}\right)^{2}}{\left(1+\mathrm{k}_{\mathrm{e}}\right)^{2}}+\frac{\left(\mathrm{L}_{\mathrm{S}_{2}}^{\mathrm{a}}\right)^{2}}{\left(1+\mathrm{k}_{\mathrm{e}}\right)^{4}}+\cdots+\frac{\left(\mathrm{L}_{\mathrm{S}_{\mathrm{N}}}^{\mathrm{a}}\right)^{2}}{\left(1+\mathrm{k}_{\mathrm{e}}\right)^{2 \mathrm{~N}}}+\frac{\left(\mathrm{L}_{\mathrm{S}_{\mathrm{TN}}}^{\mathrm{a}}\right)^{2}}{\left(1+\mathrm{k}_{\mathrm{e}}\right)^{2 \mathrm{~N}}}+2 \sum_{\mathrm{i}<\mathrm{j}} \operatorname{cov}\left[\frac{\mathrm{L}_{\mathrm{i}}^{\mathrm{a}}}{\left(1+\mathrm{k}_{\mathrm{e}}\right)^{\mathrm{i}}}, \frac{\mathrm{L}_{\mathrm{j}}^{\mathrm{a}}}{\left(1+\mathrm{k}_{\mathrm{e}}\right)^{\mathrm{j}}}\right]+ \\
& 2 \sum_{\mathrm{i}<\mathrm{N}} \operatorname{cov}\left[\frac{\mathrm{L}_{\mathrm{i}}^{\mathrm{a}}}{\left(1+\mathrm{k}_{\mathrm{e}}\right)^{\mathrm{i}}}, \frac{\mathrm{L}_{\mathrm{TN}}^{\mathrm{a}}}{\left(1+\mathrm{k}_{\mathrm{e}}\right)^{\mathrm{N}}}\right]+2 \operatorname{cov}\left[\frac{\mathrm{L}_{\mathrm{N}}^{\mathrm{a}}}{\left(1+\mathrm{k}_{\mathrm{e}}\right)^{\mathrm{N}}}, \frac{+\mathrm{L}_{\mathrm{TN}}^{\mathrm{a}}}{\left(1+\mathrm{k}_{\mathrm{e}}\right)^{\mathrm{N}}}\right]
\end{aligned}
$$

$E$, tendo em vista o conceito de coeficiente de correlação $\rho_{i j}$ aplicado aos lucros residuais $L_{i}{ }^{a}$ e $L_{j}{ }^{a}$.

$$
\rho_{\mathrm{ij}}=\frac{\operatorname{cov}\left(\mathrm{L}_{\mathrm{i}}^{\mathrm{a}}, \mathrm{L}_{\mathrm{j}}^{\mathrm{a}}\right)}{\mathrm{L}_{\mathrm{S}_{\mathrm{i}}}^{\mathrm{a}} \mathrm{L}_{\mathrm{S}_{\mathrm{j}}}^{\mathrm{a}}}
$$

vem, finalmente:

$$
\mathrm{P}_{\mathrm{S}_{0}}=\sqrt{\begin{array}{l}
\sum_{\mathrm{t}=1}^{\mathrm{N}}\left[\frac{\mathrm{L}_{\mathrm{S}_{\mathrm{t}}}^{\mathrm{a}}}{\left(1+\mathrm{k}_{\mathrm{e}}\right)^{\mathrm{t}}}\right]^{2}+\frac{\left[\mathrm{L}_{\mathrm{S}_{\mathrm{TN}}}^{\mathrm{a}}\right]^{2}}{\left(1+\mathrm{k}_{\mathrm{e}}\right)^{2 \mathrm{~N}}}+2 \sum_{\mathrm{i}<\mathrm{j}} \frac{1}{\left(1+\mathrm{k}_{\mathrm{e}}\right)^{\mathrm{i}+\mathrm{j}}} \rho_{\mathrm{ij}} \cdot \mathrm{L}_{\mathrm{S}_{\mathrm{i}}}^{\mathrm{a}} \cdot \mathrm{L}_{\mathrm{S}_{\mathrm{j}}}^{\mathrm{a}}+2 \sum_{\mathrm{i}<\mathrm{N}} \frac{1}{\left(1+\mathrm{k}_{\mathrm{e}}\right)^{\mathrm{i}+\mathrm{N}}} \rho_{\mathrm{iN}} \cdot \mathrm{L}_{\mathrm{S}_{\mathrm{i}}}^{\mathrm{a}} \cdot \mathrm{L}_{\mathrm{S}_{\mathrm{TN}}}^{\mathrm{a}} \\
\quad+\frac{2}{\left(1+\mathrm{k}_{\mathrm{e}}\right)^{2 \mathrm{~N}}} \rho_{\mathrm{N}, \mathrm{TN}} \cdot \mathrm{L}_{\mathrm{S}_{\mathrm{N}}}^{\mathrm{a}} \mathrm{L}_{\mathrm{S}_{\mathrm{TN}}}^{\mathrm{a}}
\end{array}}
$$

\subsection{Modelo de Crescimento Constante}

Partindo do pressuposto de que o lucro residual cresça a uma taxa $g$, suposta constante ao longo do tempo e compatível com a taxa de crescimento da economia, conforme Modelo de Gordon e suas limitações:

$$
\tilde{\mathrm{L}}_{\mathrm{t}}^{\mathrm{a}}=\tilde{\mathrm{L}}_{\mathrm{t}-1}^{\mathrm{a}}(1+\mathrm{g})
$$

vem 


$$
\mathrm{P}_{\mathrm{C}}=(\mathrm{PL})_{0}+\frac{\mathrm{L}_{0}^{\mathrm{a}}(1+\mathrm{g})}{\left(1+\mathrm{k}_{\mathrm{e}}\right)}+\frac{\mathrm{L}_{0}^{\mathrm{a}}(1+\mathrm{g})^{2}}{\left(1+\mathrm{k}_{\mathrm{e}}\right)^{2}}+\cdots+\frac{\mathrm{L}_{\mathrm{c}}^{\mathrm{a}}(1+\mathrm{g})^{\mathrm{n}}}{\left(1+\mathrm{k}_{\mathrm{e}}\right)^{\mathrm{n}}}
$$

Considerando que $k_{e}>g$ e assumindo $n$ muito grande, ou seja $n \rightarrow \infty$ a expressão anterior se resume a:

$$
P_{0}=(P L)_{0}+\frac{L_{1}^{a}}{k_{e}-g} \quad \text { e decorre: }
$$

- Média de $P_{0}$

$$
\mathrm{P}_{\mu_{0}}=(\mathrm{PL})_{\mathrm{c}}+\frac{\mathrm{L}_{\mu_{1}}^{\mathrm{a}}}{\mathrm{k}_{\mathrm{e}}-\mathrm{g}}
$$

Conforme DAMODARAN (1997, p. 240), embora o modelo de Gordon, neste caso aplicado aos lucros residuais, seja uma abordagem simples e interessante para a avaliação patrimonial, só pode ser usado para empresas em que a taxa de crescimento seja estável. E comenta (1997, p. 241): "Assim, embora a exigência do modelo seja para uma taxa de crescimento esperada dos dividendos, os analistas devem ser capazes de substituí-la pela taxa de crescimento nos lucros e ob-
- Desvio-Padrão de $P_{0}$

$$
\mathrm{P}_{\mathrm{S}_{0}}=\frac{\mathrm{L}_{\mathrm{S}_{1}}^{\mathrm{a}}}{\mathrm{k}_{\mathrm{e}}-\mathrm{g}}
$$

ter exatamente o mesmo resultado, caso a empresa esteja realmente num estado de equilíbrio".

\subsection{Modelo de Crescimento em Dois Estágios}

O Modelo assume dois estágios de crescimento para os lucros residuais: um estágio com taxa de crescimento alta e outro estágio subseqüente com taxa de crescimento estável. Simbolicamente:
10 estágio: taxa de crescimento $g$ por $n$ períodos taxa de desconto $\mathrm{k}_{\mathrm{e}}$ 2ºstágio: taxa de crescimento $\mathrm{g}^{*}$ estável taxa de desconto $\mathrm{k}_{\mathrm{e}}^{*}$

Logo:

$$
P_{0}=(P L)_{0}+\sum_{t=1}^{n} \frac{L_{t}^{a}}{\left(1+k_{e}\right)^{t}}+\frac{\frac{L_{n+1}^{a}}{k_{e}^{*}-g^{*}}}{\left(1+k_{e}\right)^{n}} \quad ; \operatorname{com~} k_{e}>g^{*}
$$

Decorre, então:

- Média de $P_{0}$

- Desvio-Padrão de $\mathbf{P}_{0}$

$$
\mathrm{P}_{\mu_{0}}=E\left[\mathrm{P}_{0}\right]=E\left[(P L)_{0}\right]+E\left[\frac{\mathrm{L}_{1}^{\mathrm{a}}}{\left(1+\mathrm{k}_{\mathrm{e}}\right)}+\frac{\mathrm{L}_{2}^{\mathrm{a}}}{\left(1+\mathrm{k}_{\mathrm{e}}\right)^{2}}+\cdots+\frac{\mathrm{L}_{\mathrm{n}}^{\mathrm{a}}}{\left(1+\mathrm{k}_{\mathrm{e}}\right)^{\mathrm{n}}}+\frac{\frac{\mathrm{L}_{\mathrm{n}+1}^{\mathrm{a}}}{\mathrm{k}_{\mathrm{e}}^{*}-\mathrm{g}^{*}}}{\left(1+\mathrm{k}_{\mathrm{e}}\right)^{\mathrm{n}}}\right]
$$

$$
\begin{aligned}
& P_{S_{0}}^{2}=S^{2}\left[P_{0}\right]=S^{2}\left[(P L)_{0}+\frac{L_{1}^{a}}{\left(1+k_{e}\right)}+\frac{L_{2}^{a}}{\left(1+k_{e}\right)^{2}}+\cdots+\frac{L_{n}^{a}}{\left(1+k_{e}\right)^{n}}+\left.\frac{\frac{L_{n+1}^{a}}{k_{e}^{*}-g^{*}}}{\left(1+k_{e}\right)^{n}}\right|^{t}\right] \\
& P_{\mu_{0}}=(P L)_{C}+\left[\sum_{t=1}^{n}\left(\frac{(1+g)}{\left(1+k_{e}\right)}\right)^{t} L_{\mu_{0}}^{a}+\frac{\frac{L_{\mu_{n+1}}}{k_{e}^{*}-g^{*}}}{\left(1+k_{e}\right)^{n}}\right.
\end{aligned}
$$


Decorre, então:

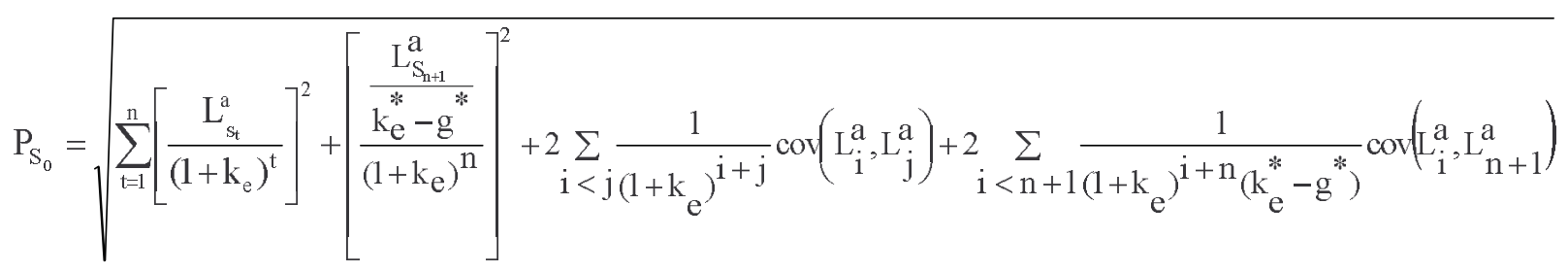

e, tendo em vista o conceito de coeficiente de correlação entre os lucros residuais, obtém-se:

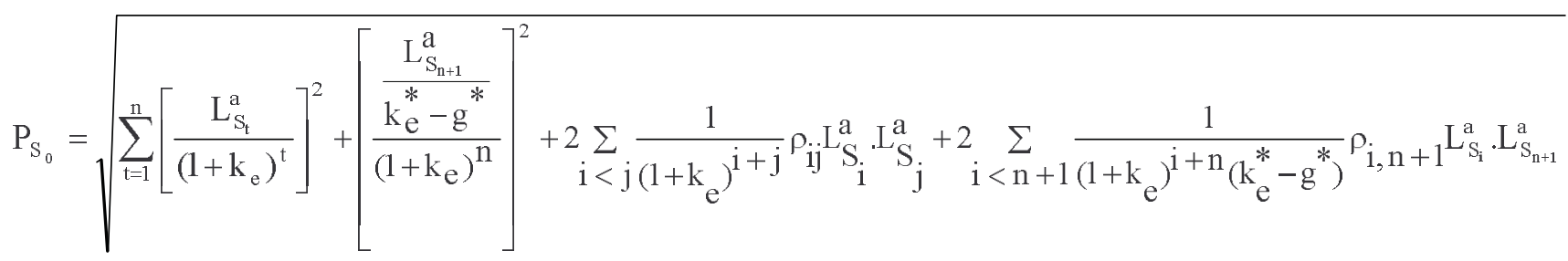

No modelo de crescimento de dois estágios, ora apresentado, o $1^{\circ}$ estágio corresponde ao período de projeção explícita e o $2^{\circ}$ estágio corresponde ao período posterior.

O valor

$$
\frac{\mathrm{L}_{\mathrm{n}+1}^{\mathrm{a}}}{\mathrm{k}_{\mathrm{e}}^{*}-\mathrm{g}^{*}}
$$

é o valor patrimonial residual ou valor terminal da empresa. Tal valor é fundamental no processo de avaliação, pois corresponde, ao ser descontado à data atual, a elevada percentagem do valor da empresa.

\section{ESTUDO EXPLORATÓRIO SOBRE A APLICABILIDADE DO MODELO DO VALOR PATRIMONIAL ALEATÓRIO}

O Estudo Exploratório realizado assumiu a premissa básica de que a aplicação do Modelo seria feita com base em dados obtidos exclusivamente de sistemas de informação consolidada, tais como LAFIS e ECONOMÁTICA, de modo a permitir ao avaliador (a) a decisão a respeito da amplitude dos dados a ser utilizados na avaliação, (b) a análise sobre eventuais ajustes, (c) estudos de sensibilidade e (d) obtenção de padrões setoriais. O Estudo Exploratório foi desenvolvido por meio de uma seqüência de passos, a seguir descrita.

\section{1 passo: Escolha das Empresas}

As empresas foram escolhidas com base nos seguintes requisitos: (a) ações da empresa negociadas na BOVESPA; (b) dados trimestrais completos dos demonstrativos contábeis da empresa - balanço patrimonial e demonstrativo do resultado do exercício - a partir do início do Plano Real; (c) possibilidade de realização de ajustes contábeis, tornando ampla a exploração metodológica pretendida; (d) distribuição constante de dividendos, de modo a respeitar as premissas do Modelo de Ohlson; (e) empresa pertencente a um segmento da economia no qual tenha havido uma variedade de negociações entre instituições, tais como fusões, aquisições e privatizações, envolvendo a necessidade de avaliações; nessas circunstâncias, o nível de informações disponíveis ao investidor permite a formação de expectativas mais acuradas e o preço das ações passa a refletir mais adequadamente o valor patrimonial de mercado da empresa.

A análise feita a partir dos parâmetros anteriores, associada às características peculiares de um estudo exploratório basicamente preocupado com a investigação metodológica, conduziu à decisão da escolha de duas empresas do setor bancário: BRADESCO e UNIBANCO.

\section{2 passo: Levantamento de Dados}

Foram coletados os seguintes dados trimestrais das empresas escolhidas: (a) lucro líquido; (b) 
patrimônio líquido; (c) saldo da conta de provisão para devedores duvidosos; (d) provisão para devedores duvidosos; (e) valor de fechamento na BOVESPA das ações preferencial e nominativa das empresas escolhidas nos dez dias úteis que envolvem o último dia útil de cada trimestre; (f) número de ações preferenciais e nominativas da empresa no último dia útil de cada trimestre; ( $\mathrm{g}$ ) taxa efetiva trimestral acumulada da taxa do CDI, escolhido como ativo livre de risco; (h) taxa efetiva trimestral do índice FGV-100, escolhido como representativo da carteira de mercado por retratar melhor em suas flutuações o desempenho geral médio da economia brasileira no período do estudo, em função de sua concentração e sua volatilidade.

\section{3 passo: Determinação do Período Histórico e Procedimentos}

Os dados trimestrais mencionados foram levantados desde junho/94, início do Plano Real, até dezembro/00. As variáveis em estudo foram focalizadas em uma janela móvel de 20 trimestres. Em seguida, o modelo foi aplicado para os 6 grupos de 20 trimestres móveis, iniciados em setembro/94 e terminados em setembro/00, e foram comparados então o valor da empresa obtido e seu valor de mercado.

\section{4 passo: As Variáveis Estudadas}

Foram estudadas as seguintes variáveis nos 6 grupos de 20 trimestres móveis para cada empresa escolhida:

- coeficiente beta: dado pelo coeficiente angular da regressão linear simples das taxas trimestrais de variação dos preços das ações em relação às taxas trimestrais de variação do índice FGV-100.

- taxa livre de risco: calculada com base na média das 20 observações trimestrais das taxas do CDI: Certificado de Depósito Bancário.

- custo do capital próprio: determinado por meio do CAPM e expresso por:

$\mathrm{k}_{\mathrm{ej}}=\mu_{\mathrm{j}(\mathrm{CDI})}+\mathrm{b}_{\mathrm{j}}\left[\mu_{\mathrm{j}(F G V-100)}-\mu_{\mathrm{j}(\mathrm{CDI})}\right] \quad ;$ onde

$\mathrm{k}_{\mathrm{ej}}=$ custo do capital próprio da empresa no grupo $j$, com $\mathrm{j}=1,6$ $\mathrm{b}_{\mathrm{j}}=$ coeficiente beta da empresa em análise no grupo $j, \operatorname{com} \mathrm{j}=1,6$.

$\mu_{\mathrm{j}(\mathrm{CDI})}=$ taxa trimestral do CDI no grupo $j$, com $\mathrm{j}=1,6$

$\mu_{\mathrm{j}(\mathrm{FGV}-100)}=$ taxa trimestral do índice FGV-100 no grupo $j$, com $\mathrm{j}=1,6$.

- Iucro residual trimestral: obtido a partir da relação:

$\mathrm{L}_{\mathrm{j}, \mathrm{t}}^{\mathrm{a}}=\mathrm{L}_{\mathrm{j}, \mathrm{t}}-\mathrm{k}_{\mathrm{ej}} \cdot(\mathrm{PL})_{\mathrm{j}, \mathrm{t}-1}(\mathrm{j}=1,6 ; \mathrm{t}=1,20)$;onde:

$\mathrm{L}_{\mathrm{j}, \mathrm{t}}^{\mathrm{a}}=$ lucro residual trimestral do trimestre $t$ no grupo $j$ $\mathrm{L}_{\mathrm{j}, \mathrm{t}}=$ lucro contábil trimestral do trimestre $t$ no grupo $j$

$(\mathrm{PL})_{\mathrm{j}, \mathrm{t}-1}=$ patrimônio líquido do trimestre $t-1$ do grupo $j$

A partir do cálculo do lucro residual trimestral das empresas escolhidas nos trimestres de cada grupo, foram estabelecidos:

$$
\mathrm{L}_{\mu_{\mathrm{j}}}^{\mathrm{a}}=\underset{\mathrm{j}=1,6}{\text { lucro residual trimestral médio no grupo } j \text {, com }}
$$
$\begin{aligned} \mathrm{L}_{\mathrm{S}_{\mathrm{j}}}^{\mathrm{a}}= & \text { desvio-padrão do lucro residual trimestral no gru- } \\ & \text { po } j, \text { com } \mathrm{j}=1,6\end{aligned}$

\section{5 passo: Determinação da taxa de crescimento $g$ do lucro residual trimestral}

O cálculo dos lucros residuais trimestrais permite estabelecer, dentro de cada grupo de trimestres, uma série histórica de lucros residuais cuja tendência (regressão linear simples) permite realizar estimativas de como seria o futuro a partir dela. Tais estimativas, entretanto, devem considerar que um observador, ao utilizar os lucros residuais trimestrais de um dado grupo; para prever como seria o futuro a partir deles, é levado a dar maior peso às informações dos trimestres que the são mais próximos, relativamente aos mais remotos.

Esse raciocínio conduz à ponderação dos lucros residuais trimestrais antes da determinação de sua tendência, isto é, regressão linear simples de lucros residuais previamente ponderados. A ponderação pode ser feita através de um modelo exponencial, conduzindo ao conhecido processo de alisamento exponencial da série histórica. $O$ coeficiente angular da regressão linear simples, assim obtida, corresponde à variação média trimestral dos lucros 
residuais no grupo de trimestres em análise. O quociente da divisão desse coeficiente angular pela média ponderada dos lucros residuais trimestrais dá uma medida do crescimento $g$ do lucro residual trimestral no interior do grupo. A extrapolação dessa medida para fora do grupo permite estimar como seriam os futuros lucros residuais.

Nestas condições, decorre:

$$
g=\frac{\text { variação média trimestral do lucro residual }}{\text { média ponderada do lucro residual }}
$$

A média ponderada do lucro residual trimestral, no grupo $j$, é calculada por:

$\left(\mathrm{L}_{\mu_{\mathrm{j}}}^{\mathrm{a}}\right)_{\text {pcnderado }}=\sum_{\mathrm{t}=1}^{20} \mathrm{p}_{\mathrm{j}, \mathrm{t}} \cdot \mathrm{L}_{\mathrm{j}, \mathrm{t}}^{\mathrm{a}}$ onde; $\quad \sum_{\mathrm{t}=1}^{2 \mathrm{C}} \mathrm{p}_{\mathrm{j}, \mathrm{t}}=1$

sendo $\mathrm{p}_{\mathrm{j}, \mathrm{t}}=$ peso do lucro residual trimestral no trimestre $t$ do grupo $j(\mathrm{t}=1,20, \mathrm{j}=1,6)$

$\operatorname{com} \mathrm{p}_{\mathrm{j}, \mathrm{t}}=\mathrm{p}_{\mathrm{j}, 1}\left(1-\mathrm{p}_{\mathrm{j}, \mathrm{t}}\right)^{\mathrm{t}-1}$

\section{6o passo: Evolução dos lucros residuais trimestrais}

Os dados coletados para o estudo exploratório, oriundos dos demonstrativos financeiros das empresas escolhidas, são trimestrais e levantados a partir do início do Plano Real, fase recente e diferenciada da economia brasileira na qual se destaca a relativa estabilidade da moeda. Os trimestres são, portanto, a unidade de medida do tempo nessa análise exploratória.

Assim sendo, o universo de dados do trabalho é composto de 27 trimestres e arranjado em 6 grupos móveis de 20 trimestres. Estendem-se para os lucros residuais trimestrais, ora em destaque, as considerações de COPELAND et al. (2000, p. 260-265), relativas ao período de projeção explícita dos fluxos de caixa líquidos, e assume-se um período de projeção explícita de 7 trimestres.

A partir da data-base, dada pelo primeiro trimestre imediatamente posterior ao término de um determinado grupo de trimestres, obtém-se a variável aleatória Valor Patrimonial da empresa expressa pelos seus

Valor Médio: $\quad \mathrm{P}_{\mu_{0}} \quad$ Desvio-Padrão: $\mathrm{P}_{\mathrm{S}_{0}}$

com base nas projeções efetuadas, e lançando mão das seguintes expressões:

\section{a) Valor Médio}

$\mathrm{P}_{\mu_{0}}=(\mathrm{PL})_{0}+\left\{\sum_{\mathrm{t}=1}^{\mathrm{n}}\left[\frac{(1+\mathrm{g})}{\left(1+\mathrm{k}_{\mathrm{e}}\right)}\right]^{\mathrm{t}}\right\} \mathrm{L}_{\mu_{0}}^{\mathrm{a}}+\frac{\frac{\mathrm{L}_{\mu_{\mathrm{n}+1}}^{\mathrm{a}}}{\mathrm{k}_{\mathrm{e}}^{*}-\mathrm{g}^{*}}}{\left(1+\mathrm{k}_{\mathrm{e}}\right)^{\mathrm{n}}} \quad ;$ onde:

$(P L)_{0}=$ patrimônio líquido da empresa na data-base.

$\mathrm{n}=$ duração do período de projeção explícita, isto é, 7 trimestres após a data-base.

$\mathrm{g}=$ taxa de crescimento do lucro residual trimestral no período de projeção explícita, obtida conforme descrito no $5^{\circ}$ passo.

$\mathrm{k}_{\mathrm{e}}$ = custo do capital próprio da empresa no período de projeção explícita, assumido igual ao custo de capital próprio do grupo de trimestres imediatamente anterior à data-base, obtido conforme descrito no $4^{\circ}$ passo.

$\mathrm{L}_{\mu \mathrm{a}}^{\mathrm{a}} \quad \mathrm{L}_{\mu \mathrm{j}}^{\mathrm{a}} \cdot(1+\mathrm{g})=$ lucro residual trimestral médio na data-base (capitalização do lucro residual trimestral médio do grupo $j$, imediatamente anterior, pela taxa $g$ ).

$\mathrm{K}_{\mathrm{e}}^{*}$ = custo de capital próprio da empresa no período posterior ao período de projeção explícita, isto é, o período perpétuo.

$\mathrm{g}^{*}=$ taxa de crescimento do lucro residual trimestral no período posterior ao período de projeção explícita, isto é, o período perpétuo.

$\mathrm{L}_{\mu_{n+1}}^{\mathrm{a}}=\mathrm{L}_{\mu_{0}}^{\mathrm{a}}(1+\mathrm{g})^{7}\left(1+\mathrm{g}^{*}\right)=$ lucro residual trimestral médio no trimestre imediatamente posterior ao período de projeção explícita. 
Para calcular o custo de capital próprio $k_{e}^{*}$ é necessário estabelecer premissas macroeconômicas para o período perpétuo de projeção, antes de se utilizar o CAPM. As premissas macroeconômicas adotadas no estudo exploratório foram estabelecidas com base no comportamento médio das variáveis presentes no CAPM nos grupos de trimestres anteriores à data-base bem como nas expectativas do futuro da economia brasileira.

No que concerne à taxa de crescimento $g^{*}$ do lucro residual no período perpétuo de projeção, também denominada taxa de crescimento estável da empresa, sua estimativa pode ser feita associando-a ao cresci- mento da economia do país em que a empresa opera, no caso, o Brasil. Conforme DAMODARAN (1997, p. 241): "Em termos práticos, a taxa de crescimento estável não pode ser maior do que a taxa nominal (real) de crescimento da economia em que a empresa opera, se a avaliação for feita em termos nominais (reais)". PINTO (2001) se refere a um estudo recente divulgado pelo BACEN, com cálculos sobre o potencial de crescimento da economia, mencionando que o potencial de crescimento até 2003 é projetado entre 3,5\% e $4,3 \%$ ao ano. Adotando essa linha de raciocínio, o estudo exploratório assumiu $\mathrm{g}^{\star}=4 \%$ a.a. para o período perpétuo de projeção.

\section{b) Desvio-Padrão}

O desvio-padrão do valor patrimonial aleatório da empresa é obtido através da relação:

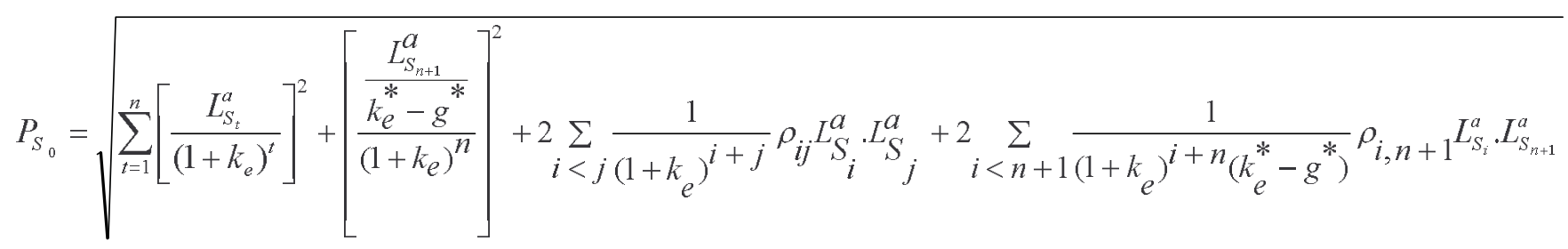

onde:

$$
\begin{aligned}
& \mathrm{L}_{\mathrm{s}_{\mathrm{t}}}^{\mathrm{a}}=\mathrm{L}_{\mathrm{S}_{0}}^{\mathrm{a}}(1+\mathrm{g})^{\mathrm{t}}=\begin{array}{l}
\text { Desvio-padrão do lucro residual trimestral no trimestre } t \text { do período de projeção } \\
\text { explícita, com } \mathrm{t}=1,7 \text {. }
\end{array} \\
& \mathrm{L}_{\mathrm{S}_{0}}^{\mathrm{a}}=\mathrm{L}_{\mathrm{S}_{\mathrm{j}}}^{\mathrm{a}}(1+\mathrm{g})=\begin{array}{l}
\text { Desvio-padrão do lucro residual trimestral na data-base (capitalização do desvio- } \\
\text { padrão do lucro residual trimestral do grupo } j \text {, imediatamente anterior, pela taxa } g \text { ) }
\end{array}
\end{aligned}
$$$$
\mathrm{L}_{\mathrm{S}_{\mathrm{n}+1}}^{\mathrm{a}}=\mathrm{L}_{\mathrm{S}_{\mathrm{n}}}^{\mathrm{a}}\left(1+\mathrm{g}^{*}\right)=\begin{aligned}
& \text { Desvio-padrão do lucro residual trimestral no trimestre imediatamente posterior } \\
& \text { ao período de projeção explícita }
\end{aligned}
$$

$\rho_{\mathrm{ij},}, \rho_{\mathrm{i}, \mathrm{n}+1}=$ coeficientes de correlação entre os lucros residuais trimestrais

As dificuldades em estabelecer os coeficientes de correlação entre os diversos pares de lucros residuais trimestrais crescem com o tamanho da duração $\mathbf{n}$ do período de projeção explícita. O número de coeficientes de correlação a serem estimados é: $n(n-1) / 2$. Para o caso de $n=27$ trimestres, o número de correlações a estimar seria de 351. Assim sendo, torna-se interessante utilizar o Modelo de Coeficiente de Correlação Único, descrito por ELTON \& GRUBER (1995, p. 128-159), que assinala a possibilidade de adotar- se um coeficiente de correlação constante entre os lucros residuais aleatórios para simplificar os procedimentos de cálculo.

Foram feitas, então, hipóteses de sensibilidade para o coeficiente de correlação, tais como:

i) $\rho_{\mathrm{ij}}=\rho=0 \quad$ ii) $\rho_{\mathrm{ij}}=\rho=1$ iii) $\rho_{\mathrm{ij}}=\rho=0,5$ e apresentados, no presente estudo, apenas os resultados que ocorreram para a hipótese $\rho_{\mathrm{ij}}=\rho=0,5$, compatível com a maior probabilidade, em termos de freqüência, de um valor intermediário positivo para correlação entre os lucros residuais. 


\section{AJUSTES REALIZADOS NO ESTUDO EXPLORATÓRIO}

O ponto-chave do modelo proposto é o conceito de lucro residual que se baseia no lucro contábil ajustado pelo custo do capital associado ao patrimônio líquido. Isso significa que a fonte básica de dados é a contabilidade da empresa, que alimenta o usuário com dados referentes a eventos passados, base para análises prospectivas. Por vezes ocorre que, para atender os seus Princípios Fundamentais, entre eles o Princípio da Prudência, a contabilidade necessita constituir ajustes. Tais ajustes têm, em geral, base estatística e podem se revelar, posteriormente, exagerados ou exíguos.

As empresas escolhidas para o Estudo Exploratório, BRADESCO e UNIBANCO, são instituições financeiras, usualmente às voltas com situações de créditos duvidosos. Neste caso, a conta de ajuste de maior relevância é a conta Provisão para Devedores Duvidosos, redutora da conta Créditos a Receber.

A constituição do fluxo de provisão para devedores duvidosos na demonstração de resultados do exercício, gerando um aumento no saldo da provisão para devedores duvidosos, afeta diretamente o lucro líquido do exercício. Posteriormente à provisão, pode ocorrer recuperação do crédito lançado como perda, bem como a ocorrência de mais perdas do que as provisionadas, caracterizando uma defasagem de reconhecimento no tempo.

A defasagem de reconhecimento no tempo das perdas de devedores duvidosos pode alterar sensivelmente o lucro líquido do exercício e, como conseqüência, o lucro residual correspondente. Essa alteração do lucro residual do exercício modifica a série histórica dos lucros residuais dos grupos de trimestres em análise, causando distorções em seus valores médios e em seus desvios-padrão respectivos. É mister, portanto, estudar índices de reversão dos créditos relativos a devedores duvidosos, o que foi feito neste trabalho, repetindo o desenvolvimento de OLIVEIRA (2001, p. 180-185).

\section{CONSIDERAÇÕES SOBRE AS TAXAS DE CRESCIMENTO DO LUCRO RESIDUAL}

\section{a) Critério de Cálculo da Taxa de Crescimento $g$ do Lucro Residual}

Uma das maiores dificuldades do estudo exploratório foi estabelecer uma metodologia de cálculo para a taxa de crescimento $\boldsymbol{g}$ do lucro residual no interior dos grupos de trimestres escolhidos. A taxa de crescimento dos lucros residuais é, sem dúvida, importante, pois permite definir a evolução dos lucros residuais futuros, peça-chave para a determinação do valor da empresa por meio do modelo ora proposto.

Os obstáculos se devem basicamente aos seguintes fatores: (i) o lucro residual é função do lucro contábil ajustado e do custo do capital próprio, e as evidências empíricas indicaram que o lucro residual, tendo em vista esses fatos, oscilava muito no interior de cada grupo de trimestres; (ii) a evolução dos lucros residuais no modelo proposto segue um regime exponencial, o que indica que a taxa de crescimento precisa considerar a variação dos lucros residuais relativamente a um lucro residual-referencial, por unidade de tempo, isto é, o trimestre; (iii) a formação de expectativas quanto aos lucros residuais futuros implica ponderação da série histórica de lucros residuais em cada grupo de trimestres.

Gerou-se, então, uma metodologia de cálculo que levasse em conta esses fatores. Fez-se o alisamento exponencial da série histórica de lucros residuais em cada grupo de trimestres e determinou-se uma tendência dos valores ponderados através de regressão linear simples. O coeficiente angular da regressão indica a variação do lucro residual por unidade de tempo. O lucro residual médio, proveniente do alisamento, passou a ser o lucro residual-referencial. O quociente da divisão do coeficiente angular da regressão pelo lucro residual médio do alisamento tornou-se a taxa de crescimento $\boldsymbol{g}$ desejada. Assim decorre:

$g=\frac{\text { coeficiente angular da regressão do alisamento }}{\text { lucro residual médio do alisamento }}$ 


\section{b) Critério de Ponderação no Alisamento Exponencial dos Lucros Residuais Históricos}

Partiu-se do princípio de que, embora o mercado não seja totalmente eficiente, os investidores possuem informações suficientemente atualizadas que permitem avaliações racionais, refletidas nos valores das ações na bolsa. Assumiu-se, então, como critério balisador da ponderação e, conseqüentemente, da taxa $g$, que o valor patrimonial de mercado se situasse, sempre que possível, no intervalo $P_{\mu} \pm P_{S}$, definido pelo modelo, em cada trimestre imediatamente anterior à data base.

Essa reflexão sobre a taxa de crescimento $\boldsymbol{g}$ permite, por meio da análise histórica, definir a estrutura mais provável de ponderadores usada pelos investidores no passado imediato e que será utilizada na data-base de interesse para a avaliação da empresa em condições de risco.

\section{Resultados do Estudo Exploratório}

O objetivo do trabalho é a proposição de um modelo aleatório de avaliação que conduz ao valor da empresa e à definição de critérios metodológicos que permitam quantificar as variáveis utilizadas no processo.

Do ponto de vista metodológico foram estabelecidos os critérios que tornaram possível quantificar:

- os coeficientes beta;

- o custo do capital próprio no período de projeção explícita e no período perpétuo;

- os lucros residuais;

- a taxa de crescimento dos lucros residuais no período de projeção explícita e no período perpétuo;

- a evolução dos lucros residuais futuros estimados;

- os coeficientes de correlação entre os lucros residuais;

- o valor de mercado;

- os ajustes ao lucro residual; e

- a estrutura de ponderação das séries históricas das variáveis utilizadas na avaliação.

O estabelecimento dos critérios quantificadores permitiu chegar aos valores em condições de risco das empresas BRADESCO e UNIBANCO nas diversas datas-base do Estudo Exploratório, por meio do modelo proposto. Os resultados desta avaliação geraram os valores patrimoniais aleatórios em cada data-base, correspondentes aos coeficientes de correlação nulo, unitário e 0,5, e foram dispostos num diagrama cartesiano. Foram plotados:

- o valor patrimonial aleatório médio (valor projetado);

- o valor patrimonial aleatório médio (valor projetado) mais um desvio-padrão;

- o valor patrimonial aleatório médio (valor projetado) menos um desvio-padrão;

- o valor patrimonial de mercado (valor ocorrido).

O gráfico do BRADESCO - fig. 1 - e o gráfico do UNIBANCO - fig. 2 - foram construídos da forma a seguir apresentada.

a) Há 6 datas-base: Setembro/99, Dezembro/99, Março/00, Junho/00, Setembro/00 e Dezembro/ 00. Para as empresas escolhidas foram feitas avaliações em cada data-base, usando o modelo desenvolvido no item 5.2 deste artigo, onde calculamos o valor patrimonial médio e o desvio-padrão. Estes cálculos foram elaborados a partir dos dados históricos disponíveis em 1 trimestre anterior à data-base em análise.

b) Os gráficos (fig. 1 e fig. 2) apresentam os valores patrimoniais médios e seus desvios projetados. Estes valores podem ser comparados com os respectivos valores patrimoniais de mercado, que realmente ocorreram na data.
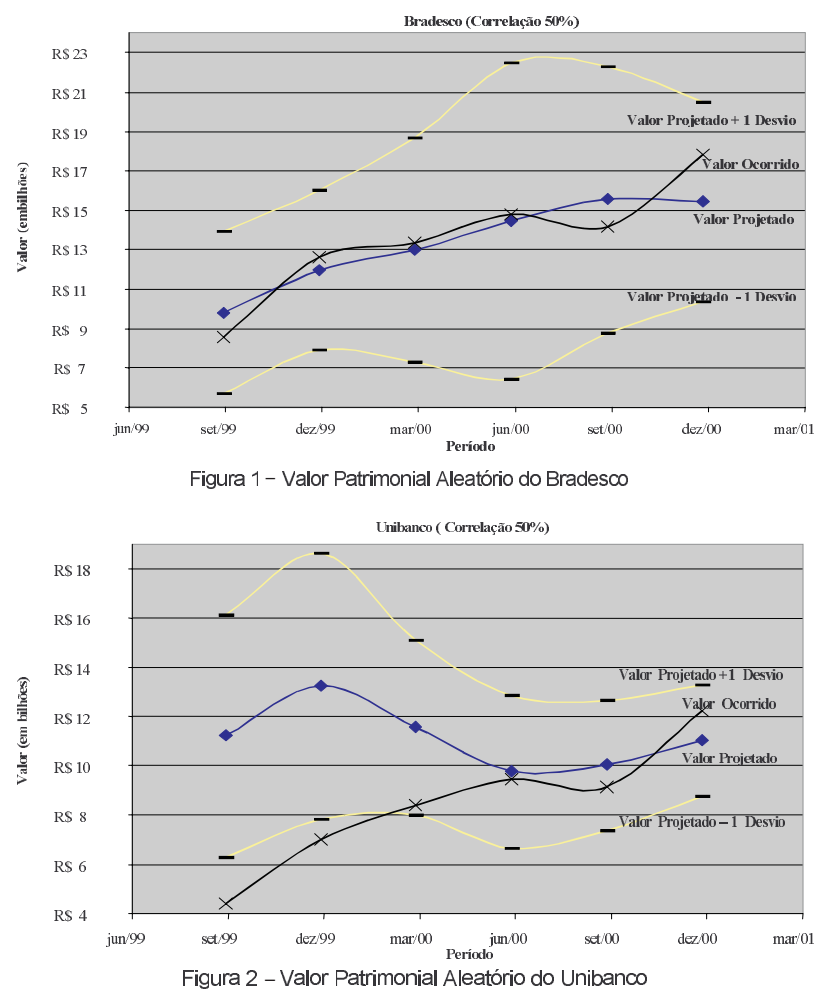
Quanto ao BRADESCO, os resultados apresentados mostram que em todas as datas-base os valores de mercado, que refletem as expectativas dos investidores quanto ao futuro da empresa, estão situados no intervalo valor médio (valor projetado) \pm 1 desvio-padrão.

No que concerne ao UNIBANCO, 2/3 dos pontos representativos dos valores de mercado nas respectivas datas-base estão situados no intervalo valor médio (valor projetado) \pm 1 desvio-padrão, sendo que, para $\pm 1,40$ desvio-padrão, todos os valores de mercado pertencem a este intervalo de confiança.

Tudo isso indica que o range de valores estabelecidos pelo modelo traduziu a convergência das avaliações dos investidores e do modelo proposto.

A análise dos resultados revela também que, à medida que os lucros residuais possuem maior correlação, as avaliações dos investidores e do mercado são ainda mais convergentes, dado que o intervalo de valores em torno da média se amplia, envolvendo os valores de mercado.

Em outras palavras, os critérios metodológicos e o modelo avaliatório se mostraram apropriados na quantificação das informações disponíveis do BRADESCO e do UNIBANCO e conduziram a avaliações consistentes com a interpretação dos investidores e suas expectativas.

\section{CONCLUSÕES}

O modelo proposto é uma extensão do modelo de Ohlson (1991), com ênfase nos aspectos aleatórios das variáveis utilizadas na avaliação, e resgata a importância das demonstrações contábeis na determinação do valor da empresa.

O valor presente dos lucros residuais futuros esperados se constitui, a rigor, em uma forma alterna- tiva de estabelecer o valor do goodwill da empresa. Este fato é, por si só, uma interessante contribuição à teoria contábil do valor.

A necessidade de ajustar o lucro líquido do período, de modo a representar adequadamente o desempenho da empresa, é um obstáculo do modelo de avaliação patrimonial aleatório pois o estudo exploratório assume que os dados contábeis sejam obtidos exclusivamente de sistemas de informação consolidada. A contribuição de Oliveira (2001: p.180185) foi decisiva no sentido de contornar esse obstáculo.

O alisamento exponencial dos lucros residuais históricos conduziu a um avanço metodológico pois permite operacionalizar a quantificação das expectativas futuras, fator preponderante de qualquer avaliação.

Os resultados da aplicação do modelo proposto às empresas escolhidas são animadores e indicam a sua potencial capacidade de predição do valor da empresa em condições de risco.

O Estudo Exploratório revelou, nos casos analisados, uma interessante convergência entre as avaliações do mercado e do modelo, dentro do intervalo de mais ou menos um desvio-padrão referenciado no valor médio (valor projetado).

No entanto, algumas dúvidas permanecem a serem dirimidas em trabalhos posteriores, relacionadas ao crescimento dos lucros residuais e ao custo do capital próprio. Modelos de crescimento com três ou mais estágios conduziriam a resultados economicamente mais interessantes? Haveria uma forma a priori de estabelecer a estrutura mais provável de ponderadores no que tange à série histórica? Que tipos de ajustes seriam necessários ao lucro contábil em empresas não-financeiras além daqueles considerados no presente trabalho? 


\section{REFERÊNCIAS BIBLIOGRÁFICAS}

COPELAND, Thomas E.; KOLLER, Tim; MURRIN, Jack. Avaliação de Empresas "Valuation": Calculando e Gerenciando o Valor das Empresas. São Paulo: Makron Books, 2000.

DAMODARAN, Aswath. Avaliação de Investimentos. Rio de Janeiro: Qualitymark, 1997.

DECHOW, Patricia M. Accounting Earnings and Cash Flows as Measures of Firm Performance: The Role of Accounting Accruals. Journal of Accounting and Economics, 18, 1995, pp. 3-42.

ELTON, Edwin; GRUBER, Martin J. Modern Portfolio Theory and Investment Analysis. $5^{\text {th }}$ ed. New York: John Wiley \& Sons, 1995.

GORDON, M.J.; SHAPIRO, E. Capital Equipment Analysis: The Required Rate of Profit. Management Science, Vol. 3, October 1956, pp. 102-110.

LOPES, Alexsandro Broedel. A relevância da informação contábil para o mercado de capitais: o modelo de Ohlson aplicado à BOVESPA. 2001. Tese (Doutorado em Controladoria e Contabilidade) - Faculdade de Economia, Administração e Contabilidade, Universidade de São Paulo, São Paulo.

MCTAGGART, James M.; KONTES, Peter W.; MANKINS, Michael C. The Value Imperative. New York: The Free Press, 1994.
O'HANLON, J.; REES, W. Links Between Accounting Numbers and Economic Fundamentals. In: REES, Bill. Financial Analysis. Hemel Hempstead: Prentice-Hall, 1995, pp. 226-254.

OHLSON, James A. The Theory of Value and Earnings, and an Introduction to the Ball-Brown Analysis. Contemporary Accounting Research, Vol.8, n.1, Fall 1991, pp. 1-19.

. Earnings, Book Values, and Dividends

in Equity Valuation. Contemporary Accounting Research, Vol. 11, n. 2, Spring 1995, pp. 661-687.

OLIVEIRA, Edson Ferreira de. Contribuição à Modelagem de Avaliação de Empresas em Condições de Risco. 2001. Tese (Doutorado em Controladoria e Contabilidade) - Faculdade de Economia, Administração e Contabilidade, Universidade de São Paulo, São Paulo.

PEASNELL, K. V. Some Formal Connection Between Economic Values and Yields and Accounting Numbers. Journal of Business Finance and Accounting, Autumn 1982, pp. 361-381.

PINTO, Celso. Pavlov e os Juros do Banco Central. Folha de S. Paulo, 22 de fevereiro de 2001, p. A-8.

STEWART, G. Bennett. The Quest for Value. USA: Harper Collins, 1991. 